\title{
EFP: NEW ENERGY-EFFICIENT FAULT-TOLERANT PROTOCOL FOR WIRELESS SENSOR NETWORK
}

\author{
Zohre Arabi ${ }^{1}$ and Roghayeh Parikhani ${ }^{2}$ \\ ${ }^{1}$ Department of Computer Engineering, Payame Noor University, PO BOX 19395-3697 \\ Tehran, IRAN \\ Zohre.arabi@gmail.com \\ ${ }^{2}$ Department of Computer Engineering, Education, meshkin shahr, IRAN \\ $r$.parikhani@gmail.com
}

\begin{abstract}
Saving energy and increasing network lifespan are important problems in wireless sensor networks (WSNs). WSNs with many small nodes can be used for monitoring and controlling the physical environments. This network due to the shared wireless environment and deployed unpleasant surroundings is fault prone. This paper introduces the New Energy-efficient Fault-tolerant Protocol for Wireless Sensor Network. Proposed EFP protocol, aims to decrease the consumption of the network resources in each round of data communication and aggregation and it is a fault tolerant technique that guarantees trustworthy of the communications between sensor nodes and base station by selecting a best sensor node as a cluster head in terms of energy and distance and electing a reserved cluster-head. Simulation results demonstrate that the suggested protocol has higher efficiency and can prolong the network lifetime.
\end{abstract}

\section{KEYWORDS}

Wireless Sensor Network, Energy efficient, fault tolerant, clustering, reserved cluster-head

\section{INTRODUCTION}

Nowadays progress in MEMS (Micro-electro-mechanical systems) and wireless network technology have made the progression of small, cheap, low power distributed devices, which are capable of local processing and wireless communication, a reality. Such devices are called wireless sensor nodes. The wireless sensor network (WSN) is a collection of small, wirelessly communicating nodes where each WSN node is equipped with several ingredients [1-3]. A diversity of sensors may be connected to the WSN node to measure attributes of phenomena. WSNs are very helpful in usages like as environmental monitoring, situation based maintenance, region monitoring, seismic monitoring and detection, martial surveillance, invoice tracking, medical monitoring and data-gathering and etc, due to their tiny size, low cost and wireless alteration. WSNs consist of several tiny, self organized nodes that form an ADHOC network that transmit sensed information to the common node which is called base station (BS) or sink. Because of the deployment of a numerous number of WSN nodes in uncontrolled or hostile environments, it is usual for the WSN nodes to grow faulty and unreliable [4-6]. Clustering is an efficient approach to decrease power consumption in the WSN. For this reason the network subdivided into smaller groups called clusters and each cluster would has a head at a time, in many cases it is referred to cluster-head $(\mathrm{CH})$, while other nodes join cluster as members. These member nodes forward sensed data to $\mathrm{CH}$ that are processed and forwarded to base station through hierarchy of CHs. A CH may be elected by the sensors of cluster or preassigned by the network designer. A $\mathrm{CH}$ is accountable for transmitting any information sensed by the WSN nodes in its cluster and can aggregate and compact the data before transmitting it to

DOI : 10.5121/ijcnc.2012.4609 
the BS. Data aggregation decreases data packet size by eliminating the information redundancy. Clustering is also useful for spatial reuse of the bandwidth due to the node clustering and robust and scalable in the face of topological changes caused by node failure, insertion or removal [7, 8]. Sensors typically consist of three main components: sensing electronics, data processing unit and communication electronics as shown in Figure. 1.

Routine clustering algorithms stand with the problem of unequal energy consumption. Clusterheads drain energy much faster than cluster members and so the network lifetime is reduced. In order to balance the energy consumption and extend network lifetime, the role of $\mathrm{CH}$ must be rotated among various members. Various designs in homogeneous sensor network are used to rotate the role of $\mathrm{CH}$ among member nodes periodically based upon some probability function. Moreover, $\mathrm{CHs}$ are also prone to hardware or software failures. To handle these faulty $\mathrm{CHs}$, some fault tolerant mechanism is required for proper functioning of the network.

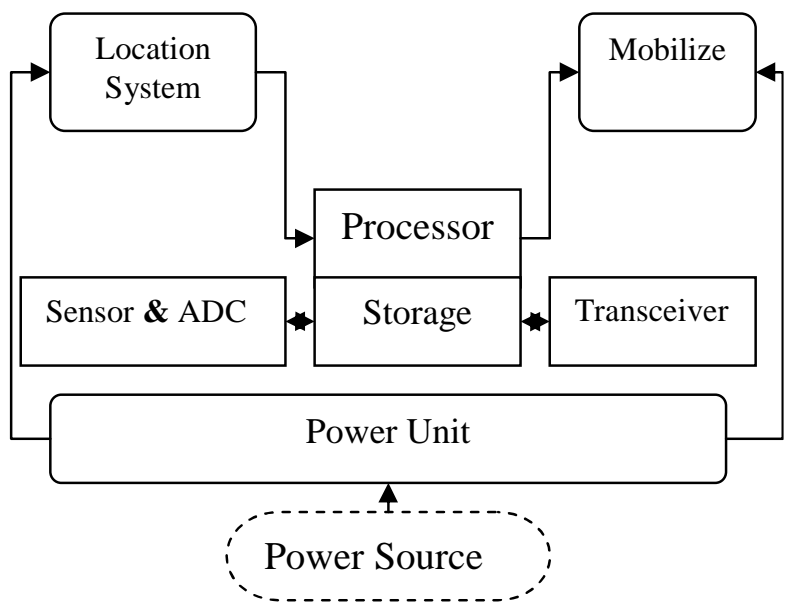

Figure 1. Components of a typical Sensor Node

In this paper, we propose and evaluate a new energy-efficient fault-tolerant (EFP) protocol for wireless sensor networks. In our considered wireless sensor network, nodes send sensing information to the cluster-head and cluster-head transmit them to base station. Certain clustering algorithms with special method periodically replacing cluster-heads and selecting reserved cluster-heads then cluster-heads aggregate the data of cluster nodes and send it to the base station. We presume that all theWSN nodes are publish homogeneously, initially all node's energy is same, any WSN nodes have limited power and the BS is fixed and not located between WSN nodes and most of the nodes are not dynamic [9].

The rest of this paper is organized as follows. In section 2, LEACH will be briefly discussed. In section 3, the proposed algorithm will be introduced. In section IV, we evaluate our mechanism compared with LEACH by simulation. Finally, in section V, we will summarize our paper and discuss about future research.

\section{Related Works}

In recent decade, a number of protocols for clustering have been suggested. In most times there are many differences between $\mathrm{CH}$ selection and clustering formation. But almost some of these clustering methods applied in homogeneous networks and not applied in heterogeneous networks. In addition, most of the usual popular clustering protocols are not fault tolerant, such as LEACH [11], PEGASIS [12] and HEED [13]. LEACH is the most popular clustering 
International Journal of Computer Networks \& Communications (IJCNC) Vol.4, No.6, November 2012

algorithm. Many of CH election protocols are based on LEACH's method. In [14] LEACH-E is proposed to select the $\mathrm{CHs}$ according to the energy remaining in each sensor node. $[15,16,17,18]$ Proposed some good energy balancing techniques.

LEACH-E protocol extend LEACH's stochastic cluster selection algorithm by a deterministic component. In LEACH-E performance like as LEACH.

The main idea in PEGASIS is form a chain among the WSN nodes so that each node will receive from and transmit to a close neighbour. Gathered data moves from node to node, get fused, and finally a designated node transmits to the BS. WSN nodes take turns sending to the BS so that the average power spent by each sensor node per round is reduced. Building a chain to minimize the total length is similar to the travelling salesman problem, which is known to be intractable. The requirement of global knowledge of the network topology makes this method difficult to implement. In the remainder of this section, we review LEACH protocol and discuss its restrictions, because LEACH is most popular in WSN clustering protocols.

\subsection{Leach (Low Energy Adaptive Clustering Hierarchy)}

In LEACH algorithm, power efficiency are reached by being $\mathrm{CH}$ in turn, and then distributing impartially the total networks energy to one WSN node, due to lowing power consuming and increasing WSN lifespan. $\mathrm{CH}$ selection depends on the whole numbers of $\mathrm{CH}$ in WSN and times that nodes have been $\mathrm{CH}$. Principles scenarios for this protocol are:

- The BS fixed in nowhere near of the nodes.

- All the WSN nodes have the same initial energy and are homogeneous in all other ways.

In first phase, algorithm chooses a node stochastically, the principal will be explained in the following: all sensor nodes compute a value $\mathrm{T}(\mathrm{n})$ according to the following formula at the beginning of all rounds:

$$
T(n)= \begin{cases}\frac{p}{1-p(r \bmod (1 / p))} & n \in G \\ 0 & \text { others }\end{cases}
$$

Where in this equation $\mathrm{P}$ describes desired percentage of $\mathrm{CHs}$ ( e.g. $\mathrm{P}=0.05$ ) current round, and $\mathrm{G}$ is the set of nodes that have not been $\mathrm{CH}$ in the last 1/P rounds.

For each node, a random number between 0 and 1 is generated. If generated number is less than $\mathrm{T}(\mathrm{n})$, this node will become a $\mathrm{CH}$ in this round and sned an advertisement message to other WSN nodes near it.

When any node has selected as $\mathrm{CH}$ itself for the current round broadcasts an advertisement message to the rest of the nodes in the network. All the non-CH nodes, after receiving this advertisement, decide on the cluster to which they will belong for this round. This decision is based on the received signal strength of the advertisement messages. After cluster head receives all the messages from the nodes that would like to be included in the cluster and based on the number of nodes in the cluster, the cluster head creates a TDMA schedule and assigns each node a time slot when it can transmit [3].

notwithstanding several benefit in using of the LEACH protocol for cluster organization, $\mathrm{CH}$ selection and incising network lifetime, there are a few features that the protocol does not backing. 
International Journal of Computer Networks \& Communications (IJCNC) Vol.4, No.6, November 2012

\section{THE EFP PROTOCOL}

In this part, the details of EFP are introduced. The main application of a WSN is to monitoring of a remote environment. Data of individual sensor nodes are almost not most important. Therefore the information of WSN nodes are associate with their neighbor nodes, data aggregation can enhancement reliability of the measured parameter and reduction the value of traffic to the BS. EFP benefits this opinion to enhancement the efficiency of the WSN. In order to develop the EFP, some assumptions are made about sensor nodes and the underlying network model. For WSN nodes, it is supposed that all nodes are can send data with enough energy to reach the BS if needed [10]. For the network, it is assumed that nodes have always data to send to the final user and the nodes located close to each other have correlated data.

like as LEACH and other LEACH based protocol, in first phase, EFP select a WSN node randomly, the main will be said in the following: all sensor nodes account a value $T(n)$ according to the following formula at the start of all rounds.

$$
T(n)=\frac{p}{1-p\left(r \bmod \left(\frac{1}{p}\right)\right)}\left[\frac{E_{n \_c u r r e n t}}{E_{n \_\max }}+\left(r_{s} \operatorname{div} \frac{1}{p}\right)\left(1-\frac{E_{n \_ \text {current }}}{E_{n \_ \text {max }}}\right)\right]
$$

In this formula $\mathrm{P}$ is the favourable percentage of $\mathrm{CHs}$ the current round, and En_current is the current power and En_max the initial power of the WSN node, with rs as the number of successive rounds in which a node has not been $\mathrm{CH}$. When rs reaches the value $1 / \mathrm{P}$ the threshold $\mathrm{T}(\mathrm{n})$ new is reset to the value it had before the inclusion of the remaining power into the threshold-equation [7]. Then, the chance of node $\mathrm{n}$ to become $\mathrm{cH}$ increases due to a higher threshold. A possible blockade of the network is solved. Additionally, rs is reset to 0 when a node becomes $\mathrm{CH}$. So, we ensure that data is transmitted to the BS as long as nodes are active [6].

After first phase and using randomization for electing the cluster-heads, algorithm compares selected cluster-head residual energy and distance between the $\mathrm{CH}$ with base station. If there is any node with more energy and less distance to BS than that node can select to cluster-head, other nodes reselect this node as main cluster-head and this node undertakes cluster-head role. In fact, after cluster formation phase, EFP protocol replaces cluster-heads based on the threshold value of residual energy and distance between the $\mathrm{CH}$ with base station on the sensor nodes based on:

$$
C(n)=\frac{E_{\text {residual }}}{d_{\text {Base-Station }}}
$$

With this method strongest node in any cluster will be selected as cluster-head and previous node as reserved one. After establishing the clusters, the steady-state phase begins and the network starts to work. But if cluster-head was damaged or corrupted, cluster members know what the cluster-head is, so they communicate with new cluster-head until the end of current round. In the next round cluster-head selection phase starts again.

Regarding to reselecting cluster-head based on residue energy and distance to BS of cluster member nodes, we can sure best node will be selected as cluster-head and the reserved clusterhead is available. This algorithm increases network lifespan and achieves fault tolerant by having double cluster-heads in each round. If the initial selected cluster-head is strongest node or its residue energy is equal to other nodes, this algorithm works like LEACH. 


\subsection{Simulation Result}

In order to measure the efficiency of the EFP protocol, the simulator, specific to the needs of our model, was coded in MATLAB.

We presume a simple model for the radio hardware power dissipation. For the simulation described here, both the free space ( $\mathrm{d} 2$ power loss) and the multi path fading (d4 power loss) channel models were used, depending on the distance between the transmitter and receiver [15]. Power control can be used to invert this loss by appropriately setting the power amplifier. If the distance is less than the threshold do, the free space (fs) model is used; otherwise, the multi path (mp) model is used. So, to transmit l-bit message a distance, the consumed energy of radio for sender is:

$$
E_{T x}(l, d)=E_{T x \text {-elec }}(l)+E_{T x \text {-amp }}(l, d)= \begin{cases}l E_{\text {elec }}+l \varepsilon f s d^{2}, & d<d_{o} \\ l E_{\text {elec }}+l \varepsilon m p d^{4}, & d \geq d_{o}\end{cases}
$$

And to receive this message, the consumed energy in receiver is:

$$
E_{R x}(l)=E_{R x-\text { elec }}(l)=l E_{\text {elec }}
$$

The electronics energy, Eelec, depends on factors such as the digital coding, modulation, filtering, and spreading of the signal, whereas the amplifier energy, efsd 2 or $\varepsilon m p d 4$, depends on the distance to the receiver and the acceptable Bit Error Rate (BER). Energy dissipation model shown in figure.2.

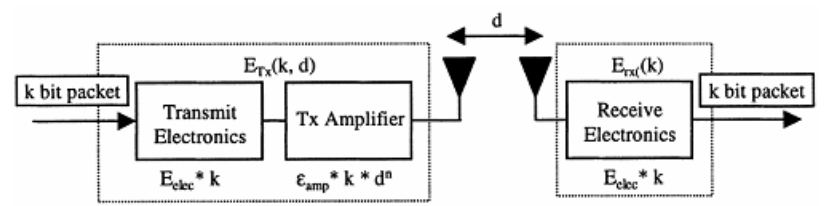

Figure 2. Radio energy dissipation model.

We compare the WSN with $\mathrm{N}=100$ nodes randomly spread in a $100 * 100 \mathrm{~m}$ surroundings. We assume that the BS fix and is located at centre of the coordinated system. The used radio parameters in our simulations are shown in Table I.

Table 1. Parameters used in simulations.

\begin{tabular}{ll}
\hline Parameter & Value \\
\hline$E_{\text {elec }}$ & $5 \mathrm{~nJ} / \mathrm{bit}$ \\
$\varepsilon £_{s}$ & $10 \mathrm{pJ} / \mathrm{bit} / \mathrm{m}^{2}$ \\
$\varepsilon_{m p}$ & $0.0013 \mathrm{pJ} / \mathrm{bit} / \mathrm{m}^{4}$ \\
$E_{0}$ & $0.3 \mathrm{~J}$ \\
$E_{D A}$ & $5 \mathrm{~nJ} / \mathrm{bit} /$ message \\
$d_{0}$ & $70 \mathrm{~m}$ \\
Message size & $8192 \mathrm{bits}$ \\
\hline
\end{tabular}

Fig. 3 present the energy consumption of the clustering protocols when the amount of nodes spread in network is 100 respectively under 2000 rounds. 
International Journal of Computer Networks \& Communications (IJCNC) Vol.4, No.6, November 2012

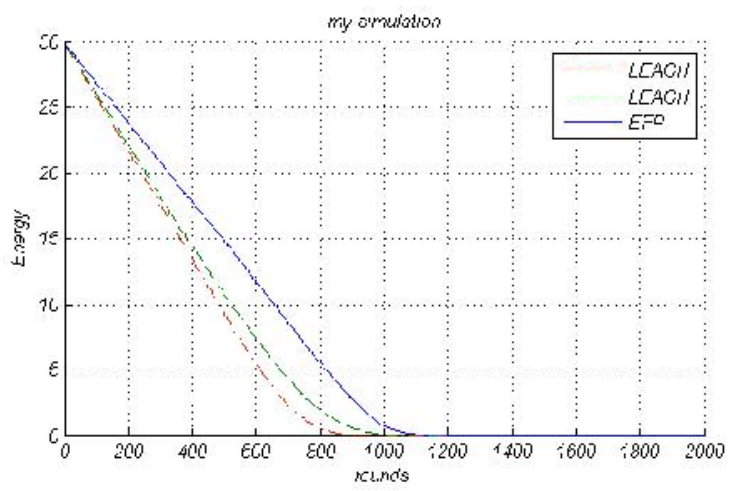

Figure 3. Total network energy with $\mathrm{N}=100$ nodes

The results demonstrate that the energy consumption of EFP protocol is generally smaller than LEACH-E and LEACH protocols.

Fig. 4 presents the number of dead nodes in the three clustering protocols. This result is closely related with the network lifetime of the wireless sensor networks.

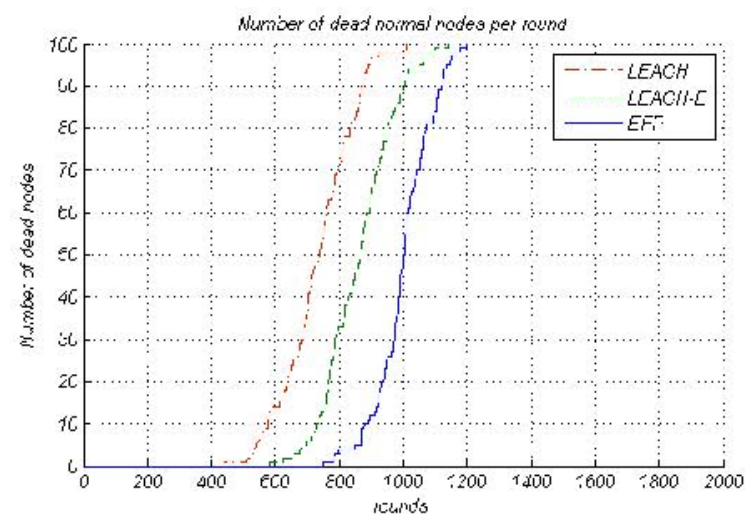

Figure 4. Total dead nodes with $\mathrm{N}=100$ nodes

Fig. 5 presents the number of alive nodes in the three clustering protocols. This result is closely related with the network lifetime of the wireless sensor networks.

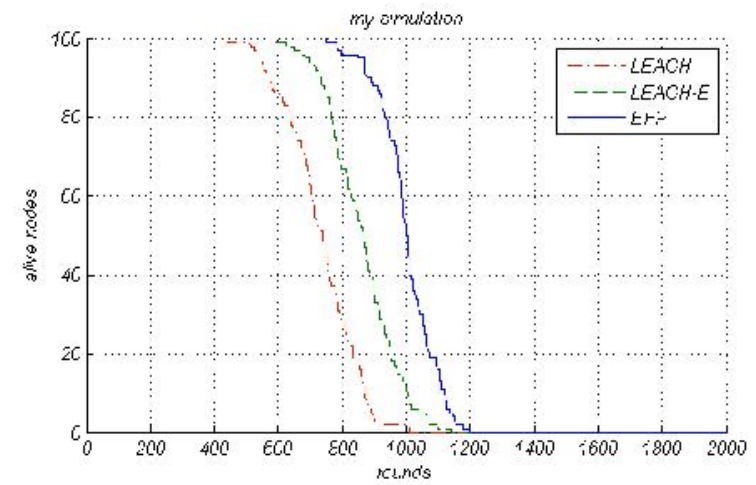

Figure 5. Total alive nodes with $\mathrm{N}=100$ nodes 
Fig. 6 present the energy consumption of the clustering protocols when the amount of nodes spread in network is 200 respectively under 1000 rounds. The $\mathrm{x}$-axis indicates the number of rounds while $y$-axis indicates the mean residual energy of each node. The results demonstrate that the energy consumption of EFP protocol is generally smaller than LEACH-E and LEACH protocols.

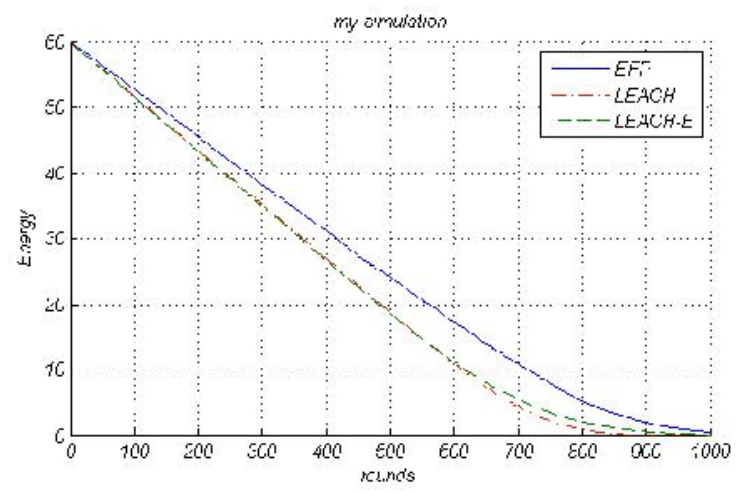

Figure 6. Total network energy with $\mathrm{N}=200$ nodes

Fig. 7 presents the number of dead nodes in the three clustering protocols with 200 nodes under 1000 rounds. This result is closely related with the network lifetime of the wireless sensor networks.

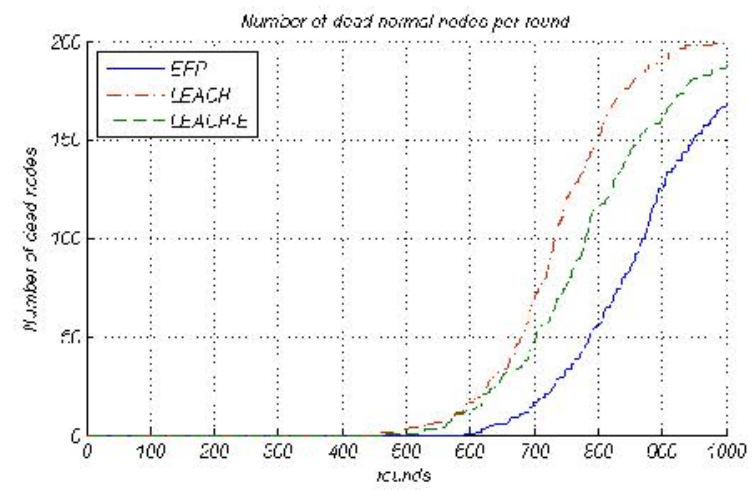

Figure 7. Total dead nodes with $\mathrm{N}=200$ nodes

The simulation results present that EFP significantly improves the lifespan and the energy consumption of the WSNs compared with other existing clustering protocols.

\section{CONCLUSIONS}

In WSNs, the power deterioration and the network lifespan are main issues for the research of the routing protocols. This paper shows EFP, a new power adaptive protocol with hierarchical clustering for WSNs that distributes loads among more powerful nodes. Proposed EFP protocol, aims to decrease the consumption of the network resources in each round of data communication and aggregation and it is a fault tolerant technique that guarantees trustworthy of the communications between sensor nodes and base station by selecting a best sensor node as a cluster head in terms of power and dimension and electing a reserved $\mathrm{CH}$. considered to the existing clustering protocols, EFP has better performance in $\mathrm{CH}$ selection and forms adaptive power impressive and adaptive clustering hierarchy. The simulation results presented that EFP 
International Journal of Computer Networks \& Communications (IJCNC) Vol.4, No.6, November 2012

improves the lifespan and the power consumption of the WSNs compared with other existing clustering protocols. Further paths of this research will be deal with clustered sensor networks with more than this parameters with in threshold calculating and more parameters to confidence value computing.

\section{REFERENCES}

[1] Gupta, G. and Younis, M. 2003.Load-Balanced Clustering in Wireless Sensor Networks. in the Proceedings of International Conference on Communication (ICC 2003), Anchorage, AK.

[2] Estrin, D., Girod, L., Pottie,G., and Srivastava, M. 2001. "Instrumenting the world with wireless sensor networks. In Proceedings of the International Conference on Acoustics, Speech and Signal Processing (ICASSP 2001). Salt Lake City, UT.

[3] Yick, J., Mukherjee, B. and Ghosal, D. 2008.Wireless sensor network survey. Computer Networks 52 (2008) 2292-2330.

[4] Biagioni, E. S. and Sasaki, G. 2003.Wireless sensor placement for reliable and efficient data collection.in the 36th International Conference on Systems Sciences, Hawaii, January.

[5] Buratti, C., Conti, A., Dardari,D. and Verdone, R. 2009.An Overview on Wireless Sensor Networks Technology and Evolution. Sensors 2009, 9, 6869-6896; doi:10.3390/s90906869.

[6] Bharathidasas, A. and Anand, V. 2002.Sensor networks: An overview. Technical report, Dept. of Computer Science, University of California at Davis.

[7] Intanagonwiwat, C., Govindan, R. and Estrin, D.2000. Directed diffusion: a scalable and robust communication paradigm for sensor networks. Mobile Computing and Networking, pp. 56-67.

[8] Krishnamachari, B., Estrin, D. and Wicker,S.B. 2002.The impact of data aggregation in wireless sensor networks. Proceedings of the 22nd International Conference on Distributed Computing Systems. (ICDCSW'02), pp. 575-578.

[9] Smaragdakis, G., Matta, I. and Bestavros, A. 2004.SEP: A Stable Election Protocol for clustered heterogeneous wireless sensor networks. Second International Workshop on Sensor and Actor Network Protocols and Applications (SANPA 2004).

[10] Heinzelman, W.R., Chandrakasan, A.P. and Balakrishnan, H. 2002.An application-specific protocol architecture for wireless microsensor net- works.IEEE Transactions on Wireless Communications 1 (4) (2002) 660-670.

[11] Lindsey, S. and Raghavenda, C.S. 2002.PEGASIS: power efficient gathering in sensor information systems. Proceeding of the IEEE Aerospace Conference, Big Sky, Montana.

[12] Younis, O. and Fahmy, S.2004.HEED: A hybrid, energy-efficient, distributed clustering approach for ad hoc sensor networks.IEEE Transactions on Mobile Computing 3 (4) (2004) 660-669.

[13] Handy, M.J., Haase, M. and Timmermann, D.2002.Low energy clustering hierarchy with deterministic cluster-head selection. Proceedings of IEEE MWCN.

[14] Rappaport, T.1996.Wireless Communications: Principles \& Practice. Englewood Cliffs, NJ: Prentice-Hall.

[15] Tandon, R., determination of optimal number of cluster inn wireless sensor networks. International Journal of Computer Networks \& Communications (IJCNC) Vol.4, No.4, July 2012

[16] Saswati, M., Matangini, Ch., Samiran, Ch., and Amrita, S., A Key Re-Distribution and Authentication Based Technique for Secured Communication in Clustered Wireless Sensor Networks with Node Mobility, International Journal of Computer Networks \& Communications (IJCNC) Vol.2, No.6, November 2010. 
International Journal of Computer Networks \& Communications (IJCNC) Vol.4, No.6, November 2012

[17] Sharma, K., Bahadur, R. Singh, P., H., A Reliable and Energy Efficient Transport Protocol for Wireless Sensor Networks, International Journal of Computer Networks \& Communications (IJCNC) Vol.2, No.5, September 2010.

[18] Sardouk, A., Rahim, R., Merghem-Boulahia, L. and Gaiti, D., Agent Strategy Data Gathering for Long WSN, International Journal of Computer Networks \& Communications (IJCNC) Vol.2, No.5, September 2010. 